\title{
A connection between state-space and doubly coprime matrix-fraction descriptions of multivariable systems
}

\author{
Chun Hsiung FANG \\ Department of Electrical Engineering, National Kaohsiung Institute of Technology, Kaohsiung 80782, Taiwan, Republic of China
}

Fan Ren CHANG

Department of Electrical Engineering, National Taiwan University, Taipei 10764, Taiwan, Republic of China

\begin{abstract}
Explicit formulas for doubly coprime matrix-fraction descriptions (MFDs) of the transfer matrix of a linear time-invariant state-space system are given in terms of a controllable and observable state-space realization of the transfer matrix. These formulas allow existing computational algorithms to be utilized for the purpose of computing doubly coprime MFDs of multivariable systems.
\end{abstract}

Keywords: Matrix-fraction description; factorization; coprime; transfer matrix.

\section{Introduction}

Over the past few decades, the MFDs approach has played an important role in linear multivariable feedback system analysis and synthesis in frequency domain [2,5,10]. However, little has been done to develop numerically feasible algorithms to compute the coprime fractional factorizations and the corresponding Bezout identity elements required for the applications of the powerful theoretical results provided by this theory [1]. A relationship between state-space representations and MFDs had been derived by Wolovich [10]. However, the state-space representation must be transformed to a controllable (observable) companion form. The tedious transformation is not needed in our method. In [6] and [9], a useful connection between state-space and doubly coprime fractional representations has been established for regular and generalized dynamical systems, respectively. However, they dealt with the problems of stable proper matrices factorizations and not polynomial matrix-fraction factorizations.

\section{Main results}

Consider a state-space system

$$
\dot{x}=A x+B u, \quad y=C x,
$$

where $A \in R^{n \times n}, B \in R^{n \times m}, C \in R^{p \times n}$ are all real matrices. The transfer matrix of the system is $T(s)=C(s I-A)^{-1} B$. Note that the system (1) here is not required to be in any canonical form. Let $\sigma$ be a real number satisfying

$$
|\sigma I+A| \neq 0
$$

and $\bar{A}, \bar{B}$ be two constant matrices defined by

$$
\bar{A}=(\sigma I+A)^{-1}, \quad \bar{B}=(\sigma I+A)^{-1} B .
$$


Lemma 1. The triple $(A, B, C)$ given in (1) is completely controllable and observable iff the triple $(\bar{A}, \bar{B}, C)$ is completely controllable and observable.

Proof. By the controllability matrix, we have

$$
\begin{aligned}
& {\left[\begin{array}{lllll}
\overline{A^{n}-1} \bar{B} & \overline{A^{n}-2} \bar{B} & \cdots & \overline{A B} & \bar{B}
\end{array}\right]} \\
& =(\sigma I+A)^{-n}\left[\begin{array}{llllll}
B & (\sigma I+A) B & \cdots & (\sigma I+A)^{n-2} B & (\sigma I+A)^{n-1} B
\end{array}\right]
\end{aligned}
$$

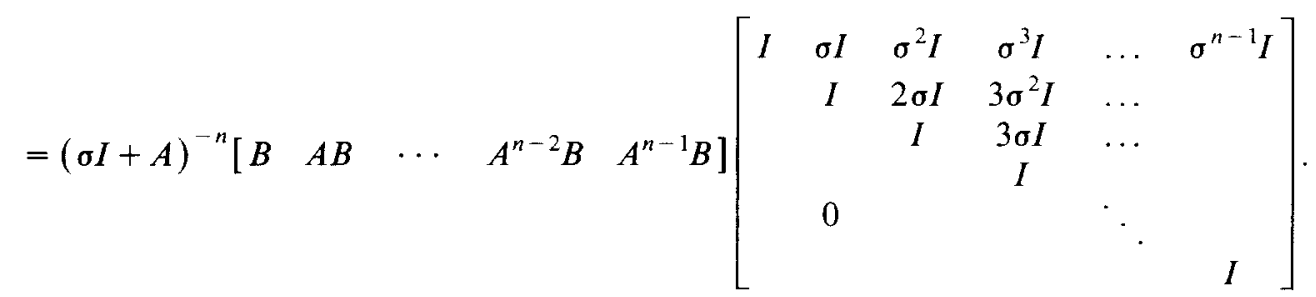

Hence the pair $(A, B)$ is completely controllable if and only if the pair $(\bar{A}, \bar{B})$ is completely controllable. The observability part can be proved in a similar way by the observability matrix.

Lemma 2 [8]. If $J$ denotes a nilpotent matrix with index $r$ then $(I-\mu J)^{-1}$ is a polynomial matrix of degree $r-1$, i.e.

$$
(I-\mu J)^{-1}=I+\mu J+\mu^{2} J^{2}+\cdots+\mu^{r-1} J^{r-1} .
$$

Our objective is to derive the doubly coprime MFDs of the transfer matrix of (1) and the corresponding elements in the generalized Bezout identity. The factorizations obtained are given in the following theorems.

Theorem 1. Given the system (1), suppose the triple $(A, B, C)$ is completely controllable and observable. Select a real number $\sigma$ satisfying (2) and choose $K \in R^{m \times n}, F \in R^{n \times p}$ such that $\bar{A}-\bar{B} K$ and $\bar{A}-F C$ are both nilpotent. Define

$$
\begin{array}{llrl}
N(\mu)=-C(I-\mu \bar{A}+\mu \bar{B} K)^{-1} \bar{B}, & D(\mu)=I-\mu K(I-\mu \bar{A}+\mu \bar{B} K)^{-1} \bar{B}, \\
\bar{X}(\mu)=-\mu^{2} K(I-\mu \bar{A}+\mu \bar{B} K)^{-1} F, & \bar{Y}(\mu)=I+\mu C(I-\mu \bar{A}+\mu \bar{B} K)^{-1} F, \\
\bar{N}(\mu)=-C(I-\mu \bar{A}+\mu F C)^{-1} \bar{B}, & \bar{D}(\mu)=I-\mu C(I-\mu \bar{A}+\mu F C)^{-1} F, \\
X(\mu)=-\mu^{2} K(I-\mu \bar{A}+\mu F C)^{-1} F, & Y(\mu)=I+\mu K(I-\mu \bar{A}+\mu F C)^{-1} \bar{B} .
\end{array}
$$

Then (a) all eight matrices described in (6) and (7) are polynomial matrices.

(b) $D(\mu)$ and $\bar{D}(\mu)$ are invertible for some $\mu$.

(c) The matrices in (6) and (7) satisfy

$$
\left[\begin{array}{cc}
\bar{D}(\mu) & \bar{N}(\mu) \\
-X(\mu) & Y(\mu)
\end{array}\right]\left[\begin{array}{cc}
\bar{Y}(\mu) & -N(\mu) \\
\bar{X}(\mu) & D(\mu)
\end{array}\right]=\left[\begin{array}{cc}
I & 0 \\
0 & I
\end{array}\right] .
$$

Proof. It is seen, from Lemma 1, that there exist two constant matrices $K \in R^{m \times n}$ and $F \in R^{n \times p}$ such that $\bar{A}-\bar{B} K$ and $\bar{A}-F C$ are both nilpotent (i.e. all eigenvalues of $\bar{A}-\bar{B} K$ and $\bar{A}-F C$ are assigned to zero). Define

$$
H(\mu)=(I-\mu \bar{A}+\mu \bar{B} K)^{-1}, \quad M(\mu)=(I-\mu \bar{A}+\mu F C)^{-1} .
$$


Lemma 2 shows that both $H(\mu)$ and $M(\mu)$ are unimodular. The identity

$$
H^{-1}(\mu)-M^{-1}(\mu)=\mu(\bar{B} K-F C)
$$

obtained from (9) will be helpful in the proof of part (c).

Part (a) is obvious from equation (9).

For (b),

$$
\begin{aligned}
\left|H^{-1}(\mu)\right||D(\mu)| & =\left|H^{-1}(\mu)\right||I-\mu K H(\mu) \bar{B}|=\left|\begin{array}{cc}
H^{-1}(\mu) & \bar{B} \\
\mu K & I
\end{array}\right| \\
& =\left|\begin{array}{cc}
I & \bar{B} \\
0 & I
\end{array}\right| \begin{array}{cc}
s I-A & 0 \\
0 & I
\end{array}|| \begin{array}{cc}
-(\sigma I+A)^{-1} & 0 \\
\mu K & I
\end{array} \mid
\end{aligned}
$$

where $s=\mu-\sigma$. Since $|s I-A| \neq 0,|\sigma I+A| \neq 0$ and $H(s)$ is unimodular, $|D(\mu)| \neq 0$. The nonsingularity of $\bar{D}(\mu)$ can be proved in a similar way.

For part (c),

$$
\begin{aligned}
\bar{N}(\mu) D(\mu) & =-C M(\mu) \bar{B}[I-\mu K H(\mu) \bar{B}]=-C M(\mu) \bar{B}+\mu C M(\mu) \bar{B} K H(\mu) \bar{B} \\
& =C M(\mu)\left[\mu \bar{B} K-H^{-1}(\mu)\right] H(\mu) \bar{B}=C M(\mu)\left[\mu F C-M^{-1}(\mu)\right] H(\mu) \bar{B} \\
& =-C H(\mu) \bar{B}+\mu C M(\mu) F C H(\mu) \bar{B}=[I-\mu C M(\mu) F][-C H(\mu) \bar{B}]=\bar{D}(\mu) N(\mu) .
\end{aligned}
$$

The readers may readily show $X(\mu) \bar{Y}(\mu)=Y(\mu) \bar{X}(\mu)$.

$$
\begin{aligned}
I-\bar{D}(\mu) \bar{Y}(\mu) & =I-[I-\mu C M(\mu) F][I+\mu C H(\mu) F] \\
& =\mu C M(\mu) F-\mu C H(\mu) F+\mu^{2} C M(\mu) F C H(\mu) F \\
& =\mu C M(\mu)\left[H^{-1}(\mu)-M^{-1}(\mu)\right] H(\mu) F+\mu^{2} C M(\mu) F C H(\mu) F \\
& =\mu^{2} C M(\mu)[\bar{B} K-F C] H(\mu) F+\mu^{2} C M(\mu) F C H(\mu) F \\
& =C M(\mu) \bar{B}\left[\mu^{2} K H(\mu) F\right] \\
& =\bar{N}(\mu) \bar{X}(\mu) .
\end{aligned}
$$

Similarly, we can show $I-Y(\mu) D(\mu)=X(\mu) N(\mu)$.

Replace the variable $\mu$ in the expressions (6) and (7) by $s+\sigma$. We then have new eight polynomial matrices:

$$
\begin{array}{llll}
N_{1}(s)=N(s+\sigma), & D_{1}(s)=D(s+\sigma), & X_{1}(s)=\bar{X}(s+\sigma), & Y_{1}(s)=\bar{Y}(s+\sigma), \\
N_{2}(s)=\bar{N}(s+\sigma), & D_{2}(s)=\bar{D}(s+\sigma), & X_{2}(s)=X(s+\sigma), & Y_{2}(s)=Y(s+\sigma) .
\end{array}
$$

The next theorem induced from Theorem 1 will present the doubly coprime matrix-fraction representations of the transfer matrix $T(s)$ of (1) and the corresponding elements in the generalized Bezout identity.

Theorem 2. The eight polynomial matrices given in (13) satisfy

$$
\begin{aligned}
& {\left[\begin{array}{cc}
D_{2}(s) & N_{2}(s) \\
-X_{1}(s) & Y_{1}(s)
\end{array}\right]\left[\begin{array}{cc}
Y_{2}(s) & -N_{1}(s) \\
X_{2}(s) & D_{1}(s)
\end{array}\right]=\left[\begin{array}{cc}
I & 0 \\
0 & I
\end{array}\right],} \\
& T(s)=N_{1}(s) D_{1}^{-1}(S)=D_{2}^{-1}(s) N_{2}(s) .
\end{aligned}
$$


Proof. (14) follows from Theorem 1 and (13).

As for (15),

$$
\begin{aligned}
N_{1}(s) D_{1}^{-1}(s) & =-C H(s+\sigma) \bar{B}[I-(s+\sigma) K H(s+\sigma) \bar{B}]^{-1} \\
& =-C H(s+\sigma)[I-(s+\sigma) \bar{B} K H(s+\sigma)]^{-1} \bar{B} \\
& =C\left[(s+\sigma) \bar{B} K-H^{-1}(s+\sigma)\right]^{-1} \bar{B}=C[(s+\sigma) \bar{A}-I]^{-1} \bar{B}=C(s I-A)^{-1} B .
\end{aligned}
$$

We can show $D_{2}^{-1}(s) N_{2}(s)=C(s I-A)^{-1} B$ in a similar fashion.

Remark 1. Theorem 2 is readily extended to the case $T(s)=C(s I-A)^{-1} B+W(s)$, where $W(s) \in R[s]^{p \times m}$ by (i) adding $W(s) D_{1}(s)$ to the expression for $N_{1}(s)$, (ii) adding $D_{2}(s) W(s)$ to the expression for $N_{2}(s)$, (iii) subtracting $X_{1}(s) W(s)$ from the expression for $Y_{1}(s)$, (iv) subtracting $W(s) X_{2}(s)$ from the expression for $Y_{2}(s)$.

Remark 2. In [10, p. 105], a nice result which provides a useful relationship between state-space and coprime matrix-fraction representations of multivariable systems has been derived. However, in contrast to ours, the controllable companion state-space representation is needed, and no expressions for the corresponding generalized Bezout identity elements are given.

Remark 3. The above theorem allows one to compute doubly coprime fractional factorizations with existing computational algorithms. Indeed, to compute the doubly coprime MFDs of the transfer matrix of the system (1) and the corresponding generalized Bezout identity elements one need only (i) determine a real number $\sigma$ satisfying (2), (ii) solve two pole-assignment (deadbeat control) problems [4,7], (iii) perform the algebraic operations given in (6), (7) and (13). Note that the computations of unimodular matrices $H(\mu)$ and $M(\mu)$ required in (6) and (7) are easy to achieve by using Lemma 2.

Remark 4. The results in Theorems 1, 2 can be extended to generalized dynamical systems. Consider a generalized dynamical system which is controllable and observable in Cobb's sense [3] and described by

$$
E \dot{x}+A x+B u, \quad y=C x
$$

where $E \in R^{n \times n}$ is a real singular matrix. The transfer matrix of (17) is $T(s)=C(s E-A)^{-1} B$. Zhou et al. [11] have shown that (17) is controllable and observable in Cobb's sense iff the triple $(\bar{A}, \bar{B}, C)$ is completely controllable and observable, where $\bar{A}, \bar{B}$ are defined as

$$
\bar{A}=(\sigma E+A)^{-1} E, \quad \bar{B}=(\sigma E+A)^{-1} B,
$$

and $\sigma$ is a real number satisfying $|\sigma E+A| \neq 0$. All results in Theorems 1,2 still hold if the definitions of $\bar{A}, \bar{B}$ given in (3) are changed to the ones in (18). If (17) is decomposed as $[3,8]$

$$
\left[\begin{array}{cc}
I_{r} & 0 \\
0 & J
\end{array}\right] \dot{x}=\left[\begin{array}{cc}
A_{1} & 0 \\
0 & I_{n-r}
\end{array}\right] x+\left[\begin{array}{l}
B_{1} \\
B_{2}
\end{array}\right] u, \quad y=\left[\begin{array}{ll}
C_{1} & C_{2}
\end{array}\right] x,
$$

where $r=\operatorname{deg}|s E-A|$ and $J$ is nilpotent, then the transfer matrix becomes $T(s)=C_{1}\left(s I_{r}-A_{1}\right)^{-1} B_{1}+$ $W_{1}(s)$, where $W_{1}(s)=C_{2}\left(s J-I_{n-r}\right)^{-1} B_{2}$ is a polynomial matrix [8]. We can then reduce it to the case considered in Remark 1.

\section{References}

[1] P.J. Antsaklis, Some relations satisfied by prime polynomial matrix and their role in linear multivariable system theory, IEEE Trans. Automat. Control 24 (1979) 611-616. 
[2] C.T. Chen, Linear System Theory and Design (Wiley, New York, 1984).

[3] D. Cobb, Controllability, observability, and duality in singular systems, IEEE Trans. Automat. Control 29 (1984) $1076-1082$.

[4] M. Gopal and P.P. Nair, On the design of a sensitivity-reducing optimal dead-beat controller, Internat. J. Control 42 (1985) 877-886.

[5] T. Kailath, Linear Systems (Prentice-Hall, Englewood Cliffs, NJ, 1980).

[6] C.N. Nett, C.A. Jacobson and M.J. Balas, A connection between state-space and doubly coprime fractional representations, IEEE Trans. Automat. Control 29 (1984) 831-832.

[7] R.V. Patel and P. Misra, Numerical algorithms for eigenvalues assignment by state feedback, Proc. IEEE 72 (1984) $1755-1764$.

[8] H.H. Rosenbrock, Structural properties of linear dynamical systems, Internat. J. Control 20 (1974) 191-202.

[9] Y.F. Wang and M.J. Balas, Doubly coprime fractional representations of generalized dynamical systems, IEEE Trans. Automat. Control 34 (1989) 733-734.

[10] W.A. Wolovich, Linear Multivariable Systems (Springer-Verlag, New York, 1974).

[11] Z. Zhou, M.A. Shayman and T.J. Tarn, Singular systems: A new approach in the time domain, IEEE Trans. Automat. Control 32 (1987) 42-50. 